\title{
Revealing the electroweak properties of a new scalar resonance
}

\section{Ian Low ${ }^{a, b}$ and Joseph Lykken ${ }^{c}$}

\author{
${ }^{a}$ High Energy Physics Division, Argonne National Laboratory, \\ Argonne, IL 60439, U.S.A. \\ ${ }^{b}$ Department of Physics and Astronomy, Northwestern University, \\ Evanston, IL 60208, U.S.A. \\ ${ }^{c}$ Fermi National Accelerator Laboratory, \\ P.O. Box 500, Batavia, IL 60510 U.S.A.
}

E-mail: ilow@anl.gov, lykken@fnal.gov

\begin{abstract}
One or more new heavy resonances may be discovered in experiments at the CERN Large Hadron Collider. In order to determine if such a resonance is the long-awaited Higgs boson, it is essential to pin down its spin, $C P$, and electroweak quantum numbers. Here we describe how to determine what role a newly-discovered neutral $C P$-even scalar plays in electroweak symmetry breaking, by measuring its relative decay rates into pairs of electroweak vector bosons: $W^{+} W^{-}, Z Z, \gamma \gamma$, and $Z \gamma$. With the data-driven assumption that electroweak symmetry breaking respects a remnant custodial symmetry, we perform a general analysis with operators up to dimension five. Remarkably, only three pure cases and one nontrivial mixed case need to be disambiguated, which can always be done if all four decay modes to electroweak vector bosons can be observed or constrained. We exhibit interesting special cases of Higgs look-alikes with nonstandard decay patterns, including a very suppressed branching to $W^{+} W^{-}$or very enhanced branchings to $\gamma \gamma$ and $Z \gamma$. Even if two vector boson branching fractions conform to Standard Model expectations for a Higgs doublet, measurements of the other two decay modes could unmask a Higgs imposter.
\end{abstract}

Keywords: Higgs Physics, Beyond Standard Model

ArXiv EPRINT: 1005.0872 


\section{Contents}

1 Introduction 1

2 Scalar couplings with $V_{1} V_{2} \quad 3$

$\begin{array}{llr}3 & \text { Implications of custodial invariance } & 6\end{array}$

4 Partial widths of $S \rightarrow V_{1} V_{2}^{(*)} \quad 10$

5 Discussion and outlook $\quad 14$

$\begin{array}{ll}\text { A General off-shell decay width } & 15\end{array}$

\section{Introduction}

Experiments at the Fermilab Tevatron and the CERN Large Hadron Collider are engaged in searches for the Higgs boson, a heavy scalar resonance predicted by the Standard Model (SM). SM Higgs bosons are excitations of the neutral $C P$-even component of an $\mathrm{SU}(2)_{L}$ weak isospin doublet field $H$ carrying unit hypercharge under $\mathrm{U}(1)_{Y}$, whose vacuum expectation value $(\mathrm{VEV}) v / \sqrt{2}$ is responsible for electroweak symmetry breaking (for reviews, see $[1,2])$.

If one or more new heavy resonances are discovered at the LHC, it will be imperative to pin down their quantum numbers relative to the expected properties of the SM Higgs. Determination of the spin and $C P$ properties of a new resonance will be challenging, although recent studies indicate that definitive results could be obtained at or around the moment of discovery, if the decay mode to $Z Z$ is observable [3-5].

Given a neutral $C P$-even spin 0 resonance $S$, one still needs to establish its electroweak quantum numbers in order to reveal any possible connection to electroweak symmetry breaking. This in turn requires information about the couplings between $S$ and pairs of vector bosons, which can be extracted from observations of $S$ decaying via $W^{+} W^{-}, Z Z$, $\gamma \gamma$, or $Z \gamma$. To an excellent approximation the couplings of the SM Higgs boson to $W W$ and $Z Z$ derive from the dimension-four Higgs kinetic terms in the SM effective action, and are thus directly related to both the strength of electroweak symmetry breaking and the electroweak quantum numbers of the Higgs field. The couplings of the SM Higgs boson to $\gamma \gamma, Z \gamma$, or a pair of gluons are elegantly derived from the observation that Higgs couplings in the SM are identical to those of a conformal-compensating dilaton in a theory where scale invariance is violated by the trace anomaly [6-9]. Thus these couplings appear first at dimension five, with coefficients related to SM gauge group beta functions.

In this paper we exhibit a general analysis, up to operators of dimension five, of the relation between the electroweak properties of $S$ and its decay branchings to $V_{1} V_{2}=W W$, 
$Z Z, \gamma \gamma$, and $Z \gamma$. We ignore decays into two gluons because of the folklore that these are unobservable, and postpone until the end a discussion of extracting complementary information from vector boson fusion production of $S[10,11]$. Nevertheless, we should emphasize that our analysis only involves the decays of the scalar into electroweak vector bosons, and hence is independent of the production mechanism of the scalar.

A key feature of our analysis is the classification of Higgs look-alikes according to the custodial symmetry $\mathrm{SU}(2)_{C}$. In the $\mathrm{SM}$ this global symmetry is the diagonal remnant after electroweak symmetry breaking of an accidental global $\mathrm{SU}(2)_{L} \times \mathrm{SU}(2)_{R}$, in which $\mathrm{SU}(2)_{L}$ and the $\mathrm{U}(1)_{Y}$ subgroup of $\mathrm{SU}(2)_{R}$ are gauged. Custodial symmetry implies $\rho \equiv$ $m_{W}^{2} /\left(m_{Z} c_{w}\right)^{2}=1[12]$, where $c_{w}$ is the cosine of the weak mixing angle. Experimentally $\rho$ is constrained to be very close to one [13], implying either that the full scalar sector respects $\mathrm{SU}(2)_{C}$, or that there are percent-level cancellations unmotivated by symmetry arguments. In our analysis we will assume that unbroken $\mathrm{SU}(2)_{C}$ is built into the scalar sector.

We consider $S$ arising from one of the neutral $C P$-even components of arbitrary spin 0 multiplets of $\mathrm{SU}(2)_{L} \times \mathrm{SU}(2)_{R}$. The case of a singlet under $\mathrm{SU}(2)_{L} \times \mathrm{SU}(2)_{R}$ is special, since then no $S V_{1} V_{2}$ couplings can appear from operators of dimension four. All other cases can be grouped according to whether the neutral scalar components transform as a singlet or a 5-plet under $\mathrm{SU}(2)_{C}$. Again under the assumption that the full scalar potential respects the custodial symmetry, these three "pure cases" only give rise to one nontrivial mixed case, i.e. when $S$ from a $\mathrm{SU}(2)_{L} \times \mathrm{SU}(2)_{R}$ singlet mixes with another $S$ from the $\mathrm{SU}(2)_{C}$ singlet part of a $\mathrm{SU}(2)_{L} \times \mathrm{SU}(2)_{R}$ nonsinglet.

Given the framework just described, we are able to enumerate all possible deviations from the SM expectations for decays of a Higgs look-alike into pairs of electroweak bosons. These deviations are typically quite large, and thus accessible to experiment at the LHC. Furthermore the deviations exhibit patterns that point towards particular non-SM scenarios. It would therefore be possible with LHC data to rule out a new scalar resonance as the agent (or the sole agent) of electroweak symmetry breaking. This possibility emphasizes the importance of observing all four $V_{1} V_{2}$ decay channels at the LHC with maximum sensitivity. We give examples of Higgs imposters that meet SM expectations for branching fractions into two of the electroweak $V_{1} V_{2}$ modes, only revealing their ersatz nature in the other two $V_{1} V_{2}$ decay modes. The approach taken here is complimentary to that in refs. [3-5], where angular correlations and total decay width were used to distinguish Higgs look-alikes. A fully global analysis using all of the available decay and production observables in each channel will of course give superior results to the simple counting experiments described here.

In section 2 we describe the dimension four couplings of an arbitrary neutral $C P$-even scalar charged under $\mathrm{SU}(2)_{L} \times \mathrm{U}(1)_{Y}$ to $W W$ or $Z Z$; we also describe the general dimension five couplings of a $\mathrm{SU}(2)_{L} \times \mathrm{U}(1)_{Y}$ singlet to two electroweak vector bosons. section 3 contains the general framework based on custodial symmetry. In section 4 we provide general results on the patterns of $S \rightarrow V_{1} V_{2}$ branching fractions, as well as discussing some interesting special cases. Further discussion and outlook are in section 5, with some general formulae for off-shell decays relegated to an appendix. 


\section{Scalar couplings with $V_{1} V_{2}$}

In this section we consider scalar couplings with two electroweak gauge bosons $V_{1} V_{2}$, where $V_{1} V_{2}=\{W W, Z Z, Z \gamma, \gamma \gamma\}$. Such couplings are dictated by the electroweak quantum numbers of the scalar $S$. We will write down $\mathrm{SU}(2)_{L} \times \mathrm{U}(1)_{Y}$ invariant operators giving rise to the $S V_{1} V_{2}$ couplings at the leading order. For an electroweak nonsinglet, the leading operator is the kinetic term of the scalar, assuming $S$ receives a VEV, while for the singlet scalar the leading operator starts at dimension five.

For nonsinglet scalars, the leading contribution to the $S V_{1} V_{2}$ coupling arises from spontaneous breaking of $\mathrm{SU}(2)_{L} \times \mathrm{U}(1)_{Y}$ down to $\mathrm{U}(1)_{e m}$ via the Higgs mechanism, when $S$ develops a VEV. It is possible to derive the general coupling when there are multiple scalars in arbitrary representations of the $\mathrm{SU}(2)_{L}$ group $[16,17]$. Using the notation $\phi_{k}$ for scalars in the complex representations and $\eta_{i}$ for scalars in the real representations, ${ }^{1}$ the kinetic terms are

$$
\sum_{k} \operatorname{Tr}\left(D_{\mu} \phi_{k}\right)^{\dagger}\left(D^{\mu} \phi_{k}\right)+\frac{1}{2} \sum_{i} \operatorname{Tr}\left(D_{\mu} \eta_{i}\right)\left(D^{\mu} \eta_{i}\right)
$$

where

$$
D_{\mu}=\partial_{\mu}-i g W_{\mu}^{a} T^{a}-\frac{i}{2} g^{\prime} B_{\mu} Y
$$

is the covariant derivative. In the above $W_{\mu}^{a}$ and $g$ are the $\mathrm{SU}(2)_{L}$ gauge bosons and gauge coupling, respectively, while $B_{\mu}$ and $g^{\prime}$ are the $\mathrm{U}(1)_{Y}$ gauge boson and gauge coupling. In addition, $T^{a}$ are the $\mathrm{SU}(2)_{L}$ generators in the corresponding representation of the scalar, and $Y$ is the hypercharge generator. For complex representations we work in the basis where $T^{3}$ and $Y$ are diagonal. After shifting the scalar fields by their VEV's: $\phi_{k} \rightarrow \phi_{k}+\left\langle\phi_{k}\right\rangle$ and $\eta_{i} \rightarrow \eta_{i}+\left\langle\eta_{i}\right\rangle$, where the VEV's are normalized as follows

$$
\operatorname{Tr}\left(\left\langle\phi_{k}\right\rangle^{\dagger}\left\langle\phi_{k}\right\rangle\right)=\frac{1}{2} v_{k}^{2}, \quad \operatorname{Tr}\left(\left\langle\eta_{i}\right\rangle^{\dagger}\left\langle\eta_{i}\right\rangle\right)=\tilde{v}_{i}^{2}
$$

electroweak symmetry is broken and $W$ and $Z$ bosons become massive. The mass eigenstates are defined as

$$
\begin{aligned}
W^{ \pm} & =\frac{1}{\sqrt{2}}\left(W^{1} \mp i W^{2}\right), \\
\left(\begin{array}{c}
W^{3} \\
B
\end{array}\right) & =\left(\begin{array}{cc}
c_{w} & s_{w} \\
-s_{w} & c_{w}
\end{array}\right)\left(\begin{array}{l}
Z \\
A
\end{array}\right),
\end{aligned}
$$

where the sine and cosine of the weak mixing angle are $c_{w}=g / \sqrt{g^{2}+g^{\prime 2}}$ and $s_{w}=$ $g^{\prime} / \sqrt{g^{2}+g^{\prime 2}}$, respectively. Notice the unbroken U(1) em leads to the conditions

$$
\left(T^{3}+\frac{1}{2} Y\right)\left\langle\phi_{k}\right\rangle=0, \quad T^{3}\left\langle\eta_{i}\right\rangle=0 .
$$

\footnotetext{
${ }^{1} \mathrm{~A}$ real representation is defined as a real multiplet with integer weak isospin and $Y=0$.
} 
Using $T^{3}\left\langle\phi_{k}\right\rangle=-Y\left\langle\phi_{k}\right\rangle / 2$ it is possible to express the mass terms of the $W$ and $Z$ in terms of the eigenvalues $T^{2}\left\langle\phi_{k}\right\rangle \equiv T^{a} T^{a}\left\langle\phi_{k}\right\rangle=T_{k}\left(T_{k}+1\right)\left\langle\phi_{k}\right\rangle$ :

$$
\begin{aligned}
m_{W}^{2} & =\frac{1}{8} g^{2} \sum_{k}\left[4 T_{k}\left(T_{k}+1\right)-Y_{k}^{2}\right] v_{k}^{2}+\frac{1}{2} g^{2} \sum_{i} T_{i}\left(T_{i}+1\right) \tilde{v}_{i}^{2}, \\
m_{Z}^{2} & =\frac{1}{4} \frac{g^{2}}{c_{w}^{2}} \sum_{k} Y_{k}^{2} v_{k}^{2},
\end{aligned}
$$

where $Y_{k}$ and $Y_{i}$ are the hypercharges of $\phi_{k}$ and $\eta_{i}$. Couplings of the real component of the neutral scalar with the $W$ and $Z$ can be read off by the replacement $v_{k} \rightarrow v_{k}\left(1+\phi_{k}^{0} / v_{k}\right)$ and $\tilde{v}_{i} \rightarrow \tilde{v}_{i}\left(1+\eta_{i}^{0} / \tilde{v}_{i}\right)$ in the mass terms:

$$
\Gamma_{S V_{1} V_{2}}^{\mu \nu}=g_{S V_{1} V_{2}} g^{\mu \nu}
$$

where $^{2}$

$$
\begin{array}{ll}
g_{\phi_{k} W W}=\frac{1}{4} g^{2}\left[4 T_{k}\left(T_{k}+1\right)-Y_{k}^{2}\right] v_{k}, & g_{\phi_{k} Z Z}=\frac{1}{2} \frac{g^{2}}{c_{w}^{2}} Y_{k}^{2} v_{k}, \\
g_{\eta_{i} W W}=g^{2} T_{i}\left(T_{i}+1\right) \tilde{v}_{i}, & g_{\eta_{i} Z Z}=0 .
\end{array}
$$

Notice that a scalar in a real representation only couples to $W W$ but not $Z Z$. Moreover, at this order there is no scalar coupling with $Z \gamma$ and $\gamma \gamma$, which are only induced at the loop level.

At this point it is worth discussing a few examples of the $\mathrm{SU}(2)_{L}$ representations appearing in the literature. The benchmark is of course the doublet Higgs scalar $H$ with $(T, Y)=(1 / 2,1)$. Couplings of the $C P$-even neutral Higgs $h$ with two electroweak bosons are

$$
g_{h W W}=\frac{1}{2} g^{2} v_{h}, \quad g_{h Z Z}=\frac{1}{2} \frac{g^{2}}{c_{w}^{2}} v_{h}, \quad g_{h Z \gamma}=g_{h \gamma \gamma}=0 .
$$

Two more popular examples are the real triplet scalar $\phi$ and the complex triplet scalar $\Phi$ with $(T, Y)=(1,0)$ and $(T, Y)=(1,2)$, respectively, for which the couplings are

$$
\begin{aligned}
& g_{\phi^{0} W W}=2 g^{2} v_{\phi}, \quad g_{\phi^{0} Z Z}=g_{\phi^{0} Z \gamma}=g_{\phi^{0} \gamma \gamma}=0, \\
& g_{\Phi^{0} W W}=g^{2} v_{\Phi}, \quad g_{\Phi^{0} Z Z}=2 \frac{g^{2}}{c_{w}^{2}} v_{\Phi}, \quad \quad g_{\Phi^{0} Z \gamma}=g_{\Phi^{0} \gamma \gamma}=0 .
\end{aligned}
$$

We see that the $S V_{1} V_{2}$ couplings are distinctly different for scalars carrying different electroweak quantum numbers, which would give rise to different patterns of decay branching ratios into two electroweak vector bosons. However, it is well known that $\phi$ and $\Phi$ individually violate the custodial symmetry and leads to unacceptably large corrections to the $\rho$ parameter unless the VEV is extremely small, on the order of a few $\mathrm{GeV}$ [13-15].

For a singlet scalar $s$, the $s V_{1} V_{2}$ couplings do not come from the Higgs mechanism. Instead, they originate from the following two dimension-five operators at the leading order:

$$
\kappa_{2} \frac{s}{4 m_{s}} W_{\mu \nu}^{a} W^{a \mu \nu}+\kappa_{1} \frac{s}{4 m_{s}} B_{\mu \nu} B^{\mu \nu},
$$

\footnotetext{
${ }^{2}$ We include a factor of 2 ! when there are two identical particles in the vertex.
} 
where the singlet $s$ is assumed to be $C P$-even. We have normalized the dimensionful couplings to the mass of the singlet $m_{s}$, although in general an unrelated mass scale could enter. In terms of the mass eigenstate in eq. (2.4), the operators become

$$
\begin{aligned}
\kappa_{2} \frac{s}{2 m_{s}} & W_{\mu \nu}^{+} W^{-\mu \nu}+\left(\kappa_{2} c_{w}^{2}+\kappa_{1} s_{w}^{2}\right) \frac{s}{4 m_{s}} Z_{\mu \nu} Z^{\mu \nu} \\
& +2 c_{w} s_{w} \frac{s}{4 m_{s}}\left(\kappa_{2}-\kappa_{1}\right) Z_{\mu \nu} F^{\mu \nu}+\left(\kappa_{2} s_{w}^{2}+\kappa_{1} c_{w}^{2}\right) \frac{s}{4 m_{s}} F_{\mu \nu} F^{\mu \nu} .
\end{aligned}
$$

from which we obtain the following couplings:

$$
\begin{aligned}
\Gamma_{s V_{1} V_{2}}^{\mu \nu} & =\frac{g_{s V_{1} V_{2}}}{m_{s}}\left(p_{V_{1}} \cdot p_{V_{2}} g^{\mu \nu}-p_{V_{1}}^{\nu} p_{V_{2}}^{\mu}\right), & & \\
g_{s W W} & =\kappa_{2}, & g_{s Z Z} & =\left(\kappa_{2} c_{w}^{2}+\kappa_{1} s_{w}^{2}\right), \\
g_{s Z \gamma} & =c_{w} s_{w}\left(\kappa_{2}-\kappa_{1}\right), & g_{s \gamma \gamma} & =\left(\kappa_{2} s_{w}^{2}+\kappa_{1} c_{w}^{2}\right) .
\end{aligned}
$$

One sees immediately that branching ratios following from these couplings are distinctly different from those coming from the Higgs mechanism. Moreover, the four couplings are controlled by only two unknown coefficients $\kappa_{2}$ and $\kappa_{1}$. So measurements of any two couplings would allow us to predict the remaining couplings, which, if verified experimentally, would be a striking confirmation of the singlet nature of the scalar resonance.

It is worth commenting that the coefficients $\kappa_{2}$ and $\kappa_{1}$ are related to the one-loop beta functions of $\mathrm{SU}(2)_{L}$ and $\mathrm{U}(1)_{Y}$ gauge groups, respectively, via the Higgs low-energy theorem $[18,19]$ :

$$
\begin{aligned}
& \beta_{2}(g)=-\frac{g^{3}}{(4 \pi)^{2}}\left(\frac{11}{3} C_{2}(G)-\frac{1}{3} n_{s} C(r)\right), \\
& \beta_{1}\left(g^{\prime}\right)=+\frac{g^{\prime 3}}{(4 \pi)^{2}} \frac{1}{3} Y^{2} n_{s}^{\prime} .
\end{aligned}
$$

In the above the Casmir invariants are defined as

$$
\operatorname{Tr}\left[t_{r}^{a} t_{r}^{b}\right]=C(r) \delta^{a b}, \quad t_{G}^{a} t_{G}^{b}=C_{2}(G) \cdot \mathbf{1}
$$

while $n_{s}$ is the number of scalars in the complex representation $r$ and $n_{s}^{\prime}$ is the number of scalars charged under $\mathrm{U}(1)_{Y}$. Such a connection has been exploited to compute that partial width of $h \rightarrow g g$ and $h \rightarrow \gamma \gamma$ in the standard model [18, 19], as well as to derive the constraints on the Higgs effective couplings [20]. For our purpose such relations serve to demonstrate that the special case of $\kappa_{2}=\kappa_{1}$, where the ratio of singlet couplings with $W W$ and $Z Z$ coincides with the standard model expectation, in general requires a conspiracy between the two one-loop beta functions to cancel each other. In this case, however, the coupling to $\gamma \gamma$ is identical to the coupling to $Z Z$. On the other hand, depending on whether the $\mathrm{SU}(2)_{L}$ running is asymptotically free, $\kappa_{2}$ and $\kappa_{1}$ could have either the same or opposite sign, resulting in a reduction (same sign) or enhancement (opposite sign) of the $Z \gamma$ width relative to $Z Z$ and $\gamma \gamma$ channels. It is also possible that $\kappa_{2}=0$, resulting in a very suppressed decay width into $W W$. We will discuss further these special cases in section 4 . 


\section{Implications of custodial invariance}

We have seen in the previous section that scalar couplings with two electroweak bosons are uniquely determined by the $\mathrm{SU}(2)_{L} \times \mathrm{U}(1)_{Y}$ quantum number of the scalar involved. For nonsinglet scalars the leading contribution to the $S V_{1} V_{2}$ couplings come from the kinetic terms via the Higgs mechanism, which in turn are related to the contribution of each scalar VEV to the masses of the $W$ and $Z$ bosons. However, the ratio of the $W$ and $Z$ masses are measured very precisely and related to the precision electroweak observable $\rho=m_{W}^{2} /\left(m_{Z} c_{w}\right)^{2}$, which is determined at the tree-level by the structure of the scalar sector in a model. Experimentally $\rho$ is very close to 1 at the percent level [13], which severely constrains the electroweak quantum number of any scalar which develops a VEV.

It has been known for a long time that the Higgs sector in the standard model possesses an accidental global symmetry $\mathrm{SU}(2)_{L} \times \mathrm{SU}(2)_{R}$, in which the $\mathrm{SU}(2)_{L}$ and $T_{R}^{3}$ are gauged and identified with the weak isospin and the hypercharge, respectively. After electroweak symmetry breaking the global symmetry is broken down to the diagonal $\mathrm{SU}(2)$, which remains unbroken. The unbroken $\mathrm{SU}(2)$ is dubbed the custodial symmetry in ref. [12], where it was shown the relation $\rho=1$ is protected by the custodial symmetry $\mathrm{SU}(2)_{C}$. In this section we classify scalar interactions with two electroweak vector bosons according to the $\mathrm{SU}(2)_{C}$ quantum number of the scalar. ${ }^{3}$

There are two possibilities for the scalar sector of a model to preserve the $\mathrm{SU}(2)_{C}$ symmetry. One could find a single irreducible representation of $\mathrm{SU}(2)_{L} \times \mathrm{U}(1)_{Y}$ which realizes $\rho=1$. In this case there is only one neutral $C P$-even scalar and the $W$ and $Z$ obtain masses from a single source, the VEV of the neutral scalar $S^{0}$. From eqs. (2.6) and (2.7) we see the condition to realize this possibility is

$$
(2 T+1)^{2}-3 Y^{2}=1 .
$$

An obvious solution is the Higgs doublet $(T, Y)=(1 / 2,1)$, beyond which the next simplest case is $(T, Y)=(3,4)[16]$. However, it is clear that, since there is only one source for the masses of $W$ and $Z$ bosons, the $S V_{1} V_{2}$ couplings are derived by replacing $m_{V} \rightarrow$ $m_{V}\left(1+S^{0} / v\right)$ in the mass term, which results in

$$
g_{S^{0} W W}=2 \frac{m_{W}^{2}}{v}, \quad g_{S^{0} Z Z}=2 \frac{m_{Z}^{2}}{v}, \quad g_{S^{0} Z \gamma}=g_{S^{0} \gamma \gamma}=0 .
$$

In other words, when there is only a single source for the mass of electroweak bosons, the custodial symmetry uniquely determines the ratio of the scalar couplings to $W W$ and $Z Z$ to be

$$
\frac{g_{S^{0} W W}}{g_{S^{0} Z Z}}=\frac{m_{W}^{2}}{m_{Z}^{2}}=c_{w}^{2},
$$

regardless of the $\mathrm{SU}(2)_{L} \times \mathrm{U}(1)_{Y}$ quantum number of the scalar involved. In the next section we will see that eq. (3.3) predicts the ratio of the decay branching fractions into $W W$ and $Z Z$ to be roughly two-to-one, which is the case in the SM with a Higgs doublet.

\footnotetext{
${ }^{3}$ In the SM the custodial invariance is explicitly broken by fermion masses, since the up-type and downtype fermions have different masses. However, this breaking is oblique in nature and only feeds into the gauge boson masses at the loop-level. Thus we do not include this particular effect in our discussion.
} 
The second possibility is to consider multiple scalars all contributing to the $W$ and $Z$ masses through the Higgs mechanism in such a way that, although individually the custodial invariance is not respected, the $\rho$ parameter remains 1 due to cancellations between the multiple scalars. This would happen if the scalars sits in a complete multiplet $\left(\mathbf{M}_{L}, \mathbf{N}_{R}\right)$ of the full $\mathrm{SU}(2)_{L} \times \mathrm{SU}(2)_{R}$ group, where $\mathbf{M}$ and $\mathbf{N}$ are positive integers labeling the $M$-dimensional and $N$-dimensional irreducible representations of $\mathrm{SU}(2)_{L}$ and $\mathrm{SU}(2)_{R}$, respectively. Recall that $\mathrm{SU}(2)_{L}$ is fully gauged and identified with the weak isospin, while $T_{R}^{3}$ is gauged and corresponds to the $\mathrm{U}(1)_{Y}$ such that $T_{R}^{3}=Y / 2$, which implies the electric charge is exactly $T_{C}^{3}$ :

$$
Q=T_{L}^{3}+\frac{Y}{2}=T_{L}^{3}+T_{R}^{3}=T_{C}^{3}
$$

Therefore, all neutral components in the scalar multiplets have $T_{C}^{3}=0$. On the other hand, unbroken custodial symmetry requires that only $\mathrm{SU}(2)_{C}$ singlets are allowed to have a VEV. In other words, the scalar representation $\left(\mathbf{M}_{L}, \mathbf{N}_{R}\right)$ must contain a state with $T_{C}=0$, where $T_{C}$ is the eigenvalue labeling the quadratic Casmir operator $T_{C}^{a} T_{C}^{a}=T_{C}\left(T_{C}+1\right) \mathbb{1}$. Since $T_{C}$ satisfies

$$
|M-N| \leq T_{C} \leq M+N
$$

we conclude that $\rho=1$ is possible only when $M=N$ and the scalar must furnish the $\left(\mathbf{N}_{L}, \mathbf{N}_{R}\right)$ representation.

The trivial representation $\left(\mathbf{1}_{L}, \mathbf{1}_{R}\right)$ is a singlet scalar under $\mathrm{SU}(2)_{L} \times \mathrm{U}(1)_{Y}$, which was considered in the previous section. In the following we focus on the non-trivial representations, in which the $S V_{1} V_{2}$ couplings arise from the Higgs mechanism after the electroweak symmetry breaking. We will represent a scalar $\Phi_{N}$ in the $\left(\mathbf{N}_{L}, \mathbf{N}_{R}\right)$ multiplet in a $N \times N$ matrix whose column vectors are $N$-plets under $\mathrm{SU}(2)_{L}$. The kinetic term of $\Phi_{N}$ is

$$
\begin{gathered}
\frac{1}{2} \operatorname{Tr}\left[\left(D^{\mu} \Phi_{N}\right)^{\dagger} D_{\mu} \Phi_{N}\right] \\
D_{\mu} \Phi_{N}=\partial_{\mu} \Phi_{N}+i g W_{\mu}^{a} T^{a} \Phi_{N}-i g^{\prime} B_{\mu} \Phi_{N} T^{3},
\end{gathered}
$$

where $T^{a}$ are generators of $\mathrm{SU}(2)$ in the $N$-plet representation. When $\Phi_{N}$ develops a VEV in a custodially invariant fashion ${ }^{4}$

$$
\left\langle\Phi_{N}\right\rangle=\frac{v}{\sqrt{2}} \mathbb{1}
$$

electroweak symmetry breaking occurs and $\rho=1$ at the tree-level.

In general various scalars in $\Phi_{N}$ could mix with one another and the mass eigenstates do not necessarily have well-defined $\mathrm{SU}(2)_{L} \times \mathrm{U}(1)_{Y}$ quantum numbers. However, it is highly desirable that the scalar potential respects the custodial symmetry so as to be consistent with $\rho=1$, which we assume to be the case. Then scalars with different $\mathrm{SU}(2)_{C}$ quantum numbers do not mix and all the mass eigenstates have definite $\mathrm{SU}(2)_{C}$ quantum numbers,

\footnotetext{
${ }^{4}$ When $N$ is an odd integer, $\Phi_{N}$ contains a real $\mathrm{SU}(2)_{L} N$-plet with zero hypercharge, whose VEV has a different normalization from that in eq. (2.3): $\tilde{v}=v / \sqrt{2}$.
} 
according to which we will proceed to classify the $S V_{1} V_{2}$ interactions. The $\left(\mathbf{N}_{L}, \mathbf{N}_{R}\right)$ representation decomposes under the unbroken $\mathrm{SU}(2)_{C}$ as

$$
\left(\mathbf{N}_{L}, \mathbf{N}_{R}\right)=\mathbf{1} \oplus \mathbf{3} \oplus \cdots \oplus \mathbf{2} \mathbf{N}-\mathbf{3} \oplus \mathbf{2} \mathbf{N}-\mathbf{1} .
$$

Scalars in the $(4 k+1)$-plet are $C P$-even and those in the $(4 k+3)$-plet are $C P$-odd. We assume no $C P$-violation in the scalar sector and neglect the $C P$-odd scalar interactions. Since we are interested in interactions with two electroweak gauge bosons, it is worth recalling that $W_{\mu}^{a}$ and $B_{\mu}$ transform as (part of) $\left(\mathbf{3}_{L}, \mathbf{3}_{R}\right)$ under $\mathrm{SU}(2)_{L} \times \mathrm{SU}(2)_{R}$. Therefore the only possible $\mathrm{SU}(2)_{C}$ quantum numbers of a system of two electroweak gauge bosons are a singlet, a triplet, or a 5-plet, which implies the scalar must also be in one of the above three representations in order to have a non-zero coupling with two electroweak bosons. We conclude that $C P$-even $S V_{1} V_{2}$ interactions are allowed only when the scalar is either a $\mathrm{SU}(2)_{C}$ singlet or a 5-plet. This is equivalent to saying two spin-1 objects can only couple to either a spin-0 or a spin-2 object. Interactions of two electroweak bosons with scalars in higher representations of $\mathrm{SU}(2)_{C}$ all vanish.

Let's define the the neutral component of a custodial $n$-plet as $H_{n}^{0}=h_{n}^{0} X_{n}^{0}$, where $h_{n}^{0}$ is the neutral scalar field and $X_{n}^{0}$ is a $N \times N$ diagonal matrix satisfying ${ }^{5}$

$$
\left[T^{a} T^{a}, X_{n}^{0}\right]=n(n+1) X_{n}^{0}, \quad\left[T^{3}, X_{n}^{0}\right]=0, \quad \operatorname{Tr}\left(X_{n}^{0} X_{n}^{0}\right)=1 .
$$

As emphasized already, only $h_{1}^{0}$ is allowed to develop a VEV. From eq. (3.8) we see that $\left\langle h_{1}^{0}\right\rangle=\sqrt{N / 2} v$ and $X_{1}^{0}=\mathbb{1} / \sqrt{N}$, which implies all other neutral components must be (diagonal) traceless matrices:

$$
\operatorname{Tr}\left(X_{n}^{0} X_{1}^{0}\right)=\operatorname{Tr}\left(X_{n}^{0}\right)=0, \quad n \geq 2 .
$$

The VEV of $h_{1}^{0}$ gives rise to the following masses from the kinetic term of $\Phi_{N}$ :

$$
\begin{aligned}
& m_{W}^{2}=\frac{1}{4} g^{2} v^{2} \operatorname{Tr}\left[T^{a} T^{a}-T^{3} T^{3}\right]=\frac{1}{24} g^{2} v^{2} N\left(N^{2}-1\right), \\
& m_{Z}^{2}=\frac{1}{2} \frac{g^{2}}{c_{w}^{2}} v^{2} \operatorname{Tr}\left[T^{3} T^{3}\right]=\frac{1}{24} \frac{g^{2}}{c_{w}^{2}} v^{2} N\left(N^{2}-1\right),
\end{aligned}
$$

which exhibits $\rho=1$. It can be verified explicitly that eqs. (3.12) and (3.13) are consistent with eqs. (2.6) and (2.7). Interactions of $h_{n}^{0}, n=1,5$, with electroweak bosons can be obtained by setting $\Phi_{N}=(v / \sqrt{2}) \mathbb{1}+H_{n}^{0}$ in eq. (3.6):

$$
\begin{aligned}
g_{h_{n}^{0} W W} & =\frac{1}{\sqrt{2}} g^{2} v \operatorname{Tr}\left[X_{n}^{0}\left(T^{a} T^{a}-T^{3} T^{3}\right)\right], \\
g_{h_{n}^{0} Z Z} & =\sqrt{2} \frac{g^{2}}{c_{w}^{2}} v \operatorname{Tr}\left[X_{n}^{0} T^{3} T^{3}\right] .
\end{aligned}
$$

For the custodial singlet, $n=1$ and $X_{1}^{0}=\mathbb{1} / \sqrt{N}$, we obtain

$$
\begin{aligned}
g_{h_{1}^{0} W W} & =\frac{1}{\sqrt{2 N}} g^{2} v \operatorname{Tr}\left[\left(T^{a} T^{a}-T^{3} T^{3}\right)\right]=2 \sqrt{\frac{2}{N}} \frac{m_{W}^{2}}{v}, \\
g_{h_{1}^{0} Z Z} & =\sqrt{\frac{2}{N}} \frac{g^{2}}{c_{w}^{2}} v \operatorname{Tr}\left[T^{3} T^{3}\right]=2 \sqrt{\frac{2}{N}} \frac{m_{Z}^{2}}{v},
\end{aligned}
$$

\footnotetext{
${ }^{5}$ Recall that neutral scalars have $T_{C}^{3}=T_{L}^{3}+T_{R}^{3}=0$ and hence belong to the diagonal entries in $\Phi_{N}$.
} 
which is a demonstration of the statement that any custodial singlet (apart from the one in the trivial representation $\left(\mathbf{1}_{L}, \mathbf{1}_{R}\right)$ ) must have couplings to the $W W$ and $Z Z$ bosons with a fixed ratio as in eq. (3.3). On the other hand, since $X_{5}^{0}$ is a traceless diagonal matrix, we have

$$
\operatorname{Tr}\left[X_{5}^{0} T^{a} T^{a}\right] \propto \operatorname{Tr}\left[X_{5}^{0} \mathbb{1}\right]=0
$$

Then the couplings are

$$
\begin{aligned}
g_{h_{5}^{0} W W} & =-\frac{1}{\sqrt{2}} g^{2} v \operatorname{Tr}\left[X_{5}^{0} T^{3} T^{3}\right], \\
g_{h_{5}^{0} Z Z} & =\sqrt{2} \frac{g^{2}}{c_{w}^{2}} v \operatorname{Tr}\left[X_{5}^{0} T^{3} T^{3}\right],
\end{aligned}
$$

which turn out to have a ratio

$$
\frac{g_{h_{5}^{0} W W}}{g_{h_{5}^{0} Z Z}}=-\frac{c_{w}^{2}}{2}
$$

that is different from the ratio of $c_{w}^{2}$ for the custodial singlet $h_{1}^{0}$. We emphasize that the ratios of the couplings only depend on the $\mathrm{SU}(2)_{C}$ quantum numbers, and not on the particular $\left(\mathbf{N}_{L}, \mathbf{N}_{R}\right)$ representation.

Again we discuss a few examples. The canonical example is the familiar Higgs doublet: $\left(\mathbf{2}_{L}, \mathbf{2}_{R}\right)=\mathbf{1} \oplus \mathbf{3}$, where the complex $\mathrm{SU}(2)_{L}$ doublet decomposes into a singlet and a triplet under $\mathrm{SU}(2)_{C}$. The $\mathrm{SU}(2)_{C}$ singlet is the neutral $C P$-even Higgs, $h$, which develops a VEV and breaks the electroweak symmetry, while the triplet contains the Goldstone bosons eaten by the $W$ and $Z$. Our general expressions in eqs. (3.16) and (3.17) are consistent with those in eq. (3.3) for $N=2$. Another example appearing in the literature [21-23] is the $\left(\mathbf{3}_{L}, \mathbf{3}_{R}\right)$ representation. Under $\mathrm{SU}(2)_{L} \times \mathrm{U}(1)_{Y}$ it consists of a real electroweak triplet with $(T, Y)=(1,0)$ and a complex electroweak triplet with $(T, Y)=(1,2)$, whose individual couplings to two electroweak bosons were summarized in eqs. (2.11) and (2.12). In this case, the $\mathrm{SU}(2)_{C}$ quantum numbers are $\left(\mathbf{3}_{L}, \mathbf{3}_{R}\right)=\mathbf{1} \oplus \mathbf{3} \oplus \mathbf{5}$, which contains two $C P$-even neutral scalars in the singlet and the 5 -plet and one $C P$-odd scalar in the triplet [21]. Our expressions for couplings of the singlet and the 5-plet with $W W$ and $Z Z$ are consistent with those in refs. [21-23]. ${ }^{6}$

It is also possible that the scalar sector of a model has multiple neutral scalar particles. In this case only scalars within the same $\mathrm{SU}(2)_{C}$ multiplet are allowed to mix in order to preserve $\rho=1$. Then the ratio of the $S V_{1} V_{2}$ couplings in the mass eigenstate depends only on the $\mathrm{SU}(2)_{C}$ quantum number and not on the mixing angle at all, except when there exists an electroweak singlet scalar $s$ which couples to $V_{1} V_{2}$ through the higher dimensional operators in eq. (2.13). In this case, it is necessary to include the loop-induced couplings of $h_{1}^{0}$ with $Z \gamma$ and $\gamma \gamma$ since they are in the same order as the $s V_{1} V_{2}$ couplings. Furthermore, there could be a higher dimensional operator of the form $s\left|D_{\mu} \Phi_{N}\right|^{2}$, with the coefficient $\kappa_{s} / m_{s}$, which gives rise to the coupling $s V_{1}^{\mu} V_{2 \mu}$ in addition to those in eq. (2.15). Even so, there are only seven unknown parameters: $g_{h_{1}^{0} W W}, g_{h_{1}^{0} Z \gamma}, g_{h_{1}^{0} \gamma \gamma}, \kappa_{1}, \kappa_{2}, \kappa_{s}$, and the mixing angle between $h_{1}^{0}$ and $s$, while one could measure a total of eight branching fractions

\footnotetext{
${ }^{6}$ Although $\rho=1$ at the tree-level in this model, constraints from $Z b \bar{b}$ vertex require $v \sim 50 \mathrm{GeV}[17]$.
} 
of two mass eigenstates decaying into $V_{1} V_{2}$. Therefore there are enough experimental measurements to not only solve for the seven unknowns, but also test the hypothesis of mixing between $h_{1}^{0}$ and $s$. If we observe multiple scalars whose couplings to two electroweak bosons do not follow from that of $h_{1}^{0}$ or $h_{5}^{0}$, one would be motivated to consider mixing of $h_{1}^{0}$ with an electroweak singlet scalar.

\section{$4 \quad$ Partial widths of $S \rightarrow V_{1} V_{2}^{(*)}$}

In this section we compute the partial decay width of $S \rightarrow V_{1} V_{2}^{(*)}$ using the couplings derived in the previous sections. Given that the mass of the scalar could be lighter than the $W W$ threshold, we include the case of $S \rightarrow V_{1} V_{2}^{*}$ when one of the vector bosons is off-shell. Although decays of an electroweak doublet scalar into two electroweak bosons have been computed both in the on-shell [24] and off-shell [25-27] cases, off-shell decays of an electroweak singlet scalar into two electroweak bosons do not appear to have been considered to the best of our knowledge. In the appendix we compute the decay width of a massive spin-0 particle into two off-shell vector bosons, which serve as the basis of the discussion in what follows.

From eq. (A.19) in the appendix decays of non-electroweak singlet scalars into $W W$ and $Z Z$ are given by

$$
\Gamma\left(S \rightarrow V_{1} V_{2}\right)=\delta_{V} \frac{1}{128 \pi} \frac{\left|\tilde{g}_{h V_{1} V_{2}}\right|^{2}}{x^{2} m_{S}} \sqrt{1-4 x}\left(1-4 x+12 x^{2}\right),
$$

where $x=m_{V}^{2} / m_{S}^{2}, \delta_{W}=2$ and $\delta_{Z}=1$. In the limit $x^{2} \ll 1$, which is a good approximation if $m_{S}$ is much larger than the $Z Z$ threshold, the pattern of a scalar decaying into two electroweak vector bosons is

$$
\Gamma(S \rightarrow W W): \Gamma(S \rightarrow Z Z): \Gamma(S \rightarrow Z \gamma): \Gamma(S \rightarrow \gamma \gamma) \approx 2 \frac{\tilde{g}_{h W W}^{2}}{m_{W}^{4}}: \frac{\tilde{g}_{h Z Z}^{2}}{m_{Z}^{4}}: 0: 0 .
$$

In terms of branching fractions, normalized to the branching ratio into $W W$, we have

$$
\begin{aligned}
B r_{S}(Z Z / W W) & =\rho^{2} c_{w}^{4} \tilde{g}_{h Z Z}^{2} / \tilde{g}_{h W W}^{2} \approx c_{w}^{4} \tilde{g}_{h Z Z}^{2} / \tilde{g}_{h W W}^{2}, \\
B r_{S}(Z \gamma / W W) & \approx B r_{S}(\gamma \gamma / W W) \approx 0,
\end{aligned}
$$

where $B r_{S}\left(V_{1} V_{2} / W W\right) \equiv \operatorname{Br}\left(S \rightarrow V_{1} V_{2}\right) / B r(S \rightarrow W W)$. Custodial symmetry then predicts unique patterns of decay branching fractions for $h_{1}^{0}$ and $h_{5}^{0}$ :

$$
\begin{array}{ll}
B r_{h_{1}^{0}}(Z Z / W W) \approx \frac{1}{2}, & B r_{h_{1}^{0}}(Z \gamma / W W) \approx B r_{h_{1}^{0}}(\gamma \gamma / W W) \approx 0, \\
B r_{h_{5}^{0}}(Z Z / W W) \approx 2, & B r_{h_{5}^{0}}(Z \gamma / W W) \approx B r_{h_{5}^{0}}(\gamma \gamma / W W) \approx 0 .
\end{array}
$$

We see that a simple counting experiment would allow us to infer the $\mathrm{SU}(2)_{C}$ quantum number of the decaying scalar!

In figure 1 we plot the ratio $\operatorname{Br}(Z Z / W W)$ for an $\mathrm{SU}(2)_{C}$ singlet and a 5-plet, including the full kinematic dependence of the gauge boson masses, for the scalar mass between 


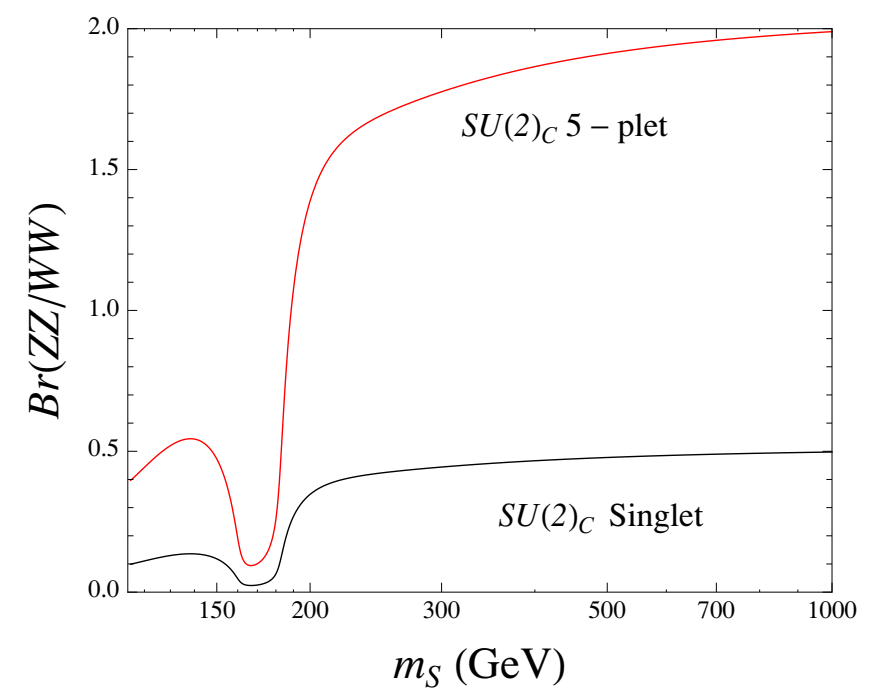

Figure 1. Ratio of branching fractions into $W W$ and $Z Z, B r(Z Z / W W)$, for an $\mathrm{SU}(2)_{C}$ singlet and a 5-plet, as a function of the scalar mass.
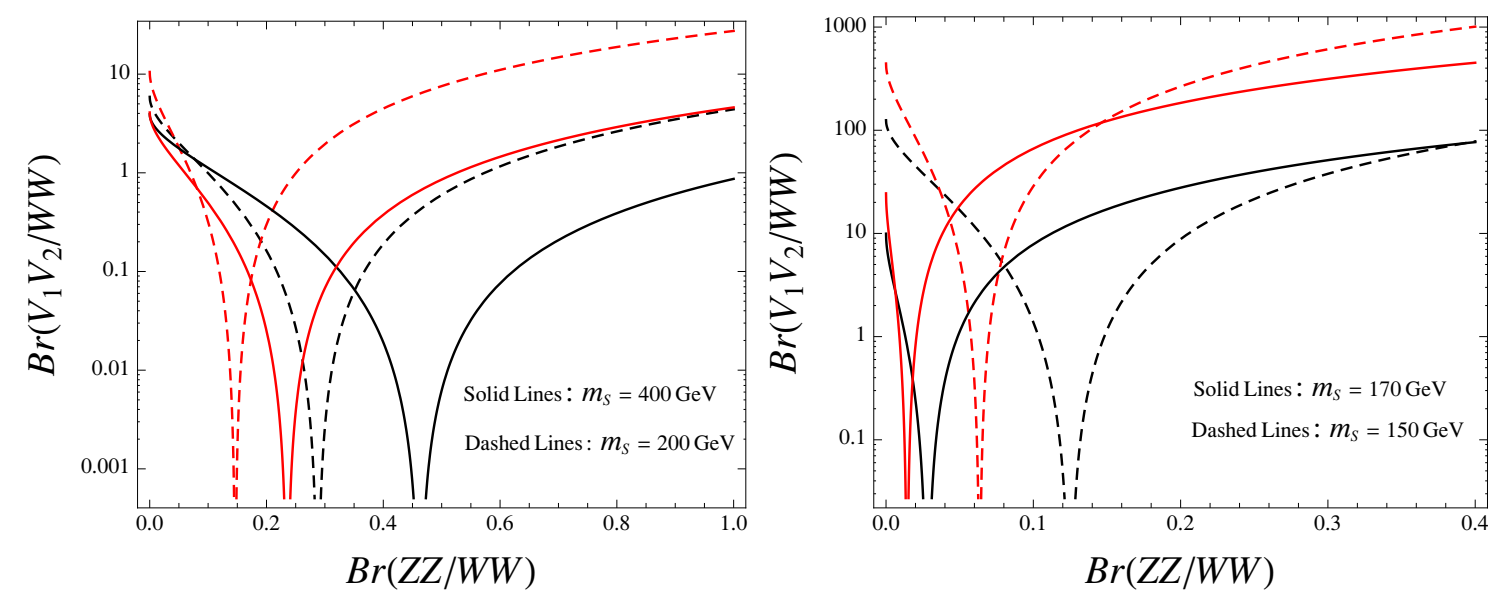

Figure 2. The predicted ratios of branchings, as a function of $B r(Z Z / W W)$, for an electroweak singlet scalar. The red (gray) curves are for $\operatorname{Br}(\gamma \gamma / W W)$ and black curves for $\operatorname{Br}(Z \gamma / W W)$. In this plot we assume the branching into $W W$ is nonzero.

$115 \mathrm{GeV}$ and $1 \mathrm{TeV}$. We include the decay into off-shell vector bosons using the expression in eq. (A.18) for the scalar mass below the $W$ and/or $Z$ threshold. Figure 1 is the unique prediction of custodial symmetry. Any deviation would imply either the electroweak singlet nature of the scalar or significant violation of custodial symmetry, which in turns suggest cancellation in the $\rho$ parameter at the percent level.

On the other hand, using eqs. (A.19), (A.22), and (A.23) in the appendix, an electroweak singlet has the following the partial decay widths into two on-shell electroweak 
bosons

$$
\begin{aligned}
\Gamma(s \rightarrow W W) & =\frac{1}{32 \pi} g_{s W W}^{2} m_{s} \sqrt{1-4 x}\left(1-4 x+6 x^{2}\right), \\
\Gamma(s \rightarrow Z Z) & =\frac{1}{64 \pi} g_{s Z Z}^{2} m_{s} \sqrt{1-4 x}\left(1-4 x+6 x^{2}\right), \\
\Gamma(s \rightarrow Z \gamma) & =\frac{1}{32 \pi} g_{s Z \gamma}^{2} m_{s}\left(1-x^{2}\right)^{3}, \\
\Gamma(s \rightarrow \gamma \gamma) & =\frac{1}{64 \pi} g_{s \gamma \gamma}^{2} m_{s},
\end{aligned}
$$

where the $g_{s V_{1} V_{2}}$ couplings are given in eq. (2.16). The pattern of partial decay widths into two electroweak bosons is then, again ignoring the effect of gauge boson masses,

$$
B r_{s}\left(V_{1} V_{2} / W W\right)=\delta_{V_{1} V_{2}} \frac{g_{s V_{1} V_{2}}^{2}}{2 g_{s W W}^{2}} .
$$

where $V_{1} V_{2}=\{Z Z, Z \gamma, \gamma \gamma\}$, and $\delta_{V_{1} V_{2}}$ is 2 for $Z \gamma$ and 1 otherwise. This pattern is generically different from that in eq. (4.2), where the couplings arise from the Higgs mechanism. More importantly, there are only two unknowns $\kappa_{1}$ and $\kappa_{2}$. So the branching fractions into $Z \gamma$ and $\gamma \gamma$, normalized to $W W$ mode, could be predicted as follows:

$$
\begin{aligned}
B r_{s}(Z \gamma / W W) & \approx \frac{c_{w}^{2}}{s_{w}^{2}}\left[\sqrt{2 B r_{s}(Z Z / W W)}-1\right]^{2} \\
B r_{s}(\gamma \gamma / W W) & \approx \frac{1}{2}\left[\frac{c_{w}^{2}}{s_{w}^{2}} \sqrt{2 B r_{s}(Z Z / W W)}+1-\frac{c_{w}^{2}}{s_{w}^{2}}\right]^{2} .
\end{aligned}
$$

In figure 2 we plot the predicted $B r(Z \gamma / W W)$ and $B r(\gamma \gamma / W W)$ branching fractions in terms of $\operatorname{Br}(Z Z / W W)$. Experimental verification of these relations would be a striking confirmation of the singlet nature of the scalar resonance.

By inspection of eq. (2.16) we see that a special case occurs when $\kappa_{2}=\kappa_{1}$, giving $B r_{s}(Z Z / W W)=1 / 2$, similar to that of $h_{1}^{0}$. However, in this case we have

$$
B r_{s}(Z \gamma / W W) \approx 0, \quad B r_{s}(\gamma \gamma / W W) \approx \frac{1}{2},
$$

up to corrections due to the mass of the $Z$ boson. By considering all four partial widths into the electroweak bosons it is still possible to distinguish a singlet scalar from the Higgs doublet even in this special case. However, as commented in the end of section 2, such a scenario lacks any obvious physical motivation.

Another special case is when $\kappa_{1}=0$, which occurs in the event that the new fermions inducing the dimension-five operators in eq. (2.13) carry only hypercharge and no isospin. This case is not included in figure 2 since the partial width of the scalar decaying into $W W$ vanishes! Nevertheless, there would still be significant decay branching fractions into $Z Z$, $Z \gamma$, and $\gamma \gamma$ states, as predicted by eq. (2.16).

In table I we list the ratios of branching fractions for an electroweak singlet, when $B r_{s}(Z Z / W W)$ of the scalar is "tuned" to fake that of a SM Higgs doublet. We see in all cases $B r_{s}(Z \gamma / W W)$ and $B r_{s}(\gamma \gamma / W W)$ are enhanced over that of the SM ratios, 


\begin{tabular}{|c|c|c|c|}
\hline$m_{S}(\mathrm{GeV})$ & $B r(Z Z / W W)$ & $B r(Z \gamma / W W)$ & $B r(\gamma \gamma / W W)$ \\
\hline 130 & $0.13(0.13)$ & $4.3 \times 10^{-2}\left(6.7 \times 10^{-3}\right)$ & $3.8 \times 10^{2}\left(7.8 \times 10^{-3}\right)$ \\
\hline 150 & $0.12(0.12)$ & $1.9 \times 10^{-2}\left(3.5 \times 10^{-3}\right)$ & $65\left(2.0 \times 10^{-3}\right)$ \\
\hline 170 & $2.3 \times 10^{-2}\left(2.3 \times 10^{-2}\right)$ & $7.8 \times 10^{-2}\left(4.1 \times 10^{-4}\right)$ & $1.9\left(1.6 \times 10^{-4}\right)$ \\
\hline 200 & $0.36(0.36)$ & $7.3 \times 10^{-2}\left(2.4 \times 10^{-4}\right)$ & $3.3\left(\gtrsim 10^{-4}\right)$ \\
\hline 300 & $0.44(0.44)$ & $1.1 \times 10^{-3}\left(\gtrsim 10^{-4}\right)$ & $0.91\left(\gtrsim 10^{-4}\right)$ \\
\hline 400 & $0.47(0.47)$ & $\gtrsim 10^{-4}\left(\gtrsim 10^{-4}\right)$ & $0.68\left(\gtrsim 10^{-4}\right)$ \\
\hline
\end{tabular}

Table 1. Ratios of branching fractions for an electroweak singlet scalar when $\operatorname{Br}(Z Z / W W)$ is tuned to the SM value. The value in the parenthesis is for the corresponding SM prediction.

\begin{tabular}{|c|c|c|c|}
\hline$m_{S}(\mathrm{GeV})$ & $\operatorname{Br}(\gamma \gamma / W W)$ & $B r(Z Z / W W)$ & $\operatorname{Br}(Z \gamma / W W)$ \\
\hline 115 & $2.7 \times 10^{-2}\left(2.7 \times 10^{-2}\right)$ & $5.1 \times 10^{-2}(0.11)$ & $39\left(9.0 \times 10^{-3}\right)$ \\
\hline 120 & $1.7 \times 10^{-2}\left(1.7 \times 10^{-2}\right)$ & $5.7 \times 10^{-2}(0.11)$ & $35\left(8.2 \times 10^{-3}\right)$ \\
\hline 130 & $7.8 \times 10^{-3}\left(7.8 \times 10^{-3}\right)$ & $6.7 \times 10^{-2}(0.13)$ & $26\left(6.7 \times 10^{-3}\right)$ \\
\hline 140 & $4.0 \times 10^{-3}\left(4.0 \times 10^{-3}\right)$ & $7.1 \times 10^{-2}(0.14)$ & $18\left(5.1 \times 10^{-3}\right)$ \\
\hline 150 & $2.0 \times 10^{-3}\left(2.0 \times 10^{-3}\right)$ & $6.4 \times 10^{-2}(0.12)$ & $10\left(3.5 \times 10^{-3}\right)$ \\
\hline 170 & $1.6 \times 10^{-4}\left(1.6 \times 10^{-4}\right)$ & $1.4 \times 10^{-2}\left(2.3 \times 10^{-2}\right)$ & $0.81\left(4.1 \times 10^{-4}\right)$ \\
\hline
\end{tabular}

Table 2. Ratios of branching fractions for an electroweak singlet scalar when $\operatorname{Br}(\gamma \gamma / W W)$ is tuned to the SM value. The value in the parenthesis is for the corresponding SM prediction.

especially in the low mass region, when the difference could reach five orders of magnitude at $m_{S}=130 \mathrm{GeV}$ for $B r_{s}(\gamma \gamma / W W)$. The reason behind the enhancement is quite easy to understand: the singlet coupling strengths to all four vector boson pairs are all in the same order. Thus decays into massive final states such as $Z Z$ and $W W$ are suppressed due to phase space and kinematic factors, especially in the low scalar mass region when $W W$ and $Z Z$ channels are off-shell. To the contrary, in the SM the Higgs couplings to $W W$ and $Z Z$ arise at the tree-level while the couplings to $Z \gamma$ and $\gamma \gamma$ come from dimension-five operators at the one-loop level. So decays into massive final states could still dominate even below the kinematic threshold.

Another interesting case is exhibited in table II, where $B r_{s}(\gamma \gamma / W W)$ is dialed to fake that of the SM Higgs. In this case the $Z Z$ channel is suppressed relative to the $W W$ channel, while the $Z \gamma$ channel is significantly enhanced. The importance of $Z \gamma$ decays is notable, since this channel is so far neglected in the physics planning of the LHC experiments.

If one makes the assumption that the individual partial decay width of a scalar decaying into to $V_{1} V_{2}$ could be obtained, presumably in a future lepton collider or with a very high integrated luminosity at the LHC, then we could explore the possibility of determining the $\left(\mathbf{N}_{L}, \mathbf{N}_{R}\right)$ multiplet structure under $\mathrm{SU}(2)_{L} \times \mathrm{SU}(2)_{R}$. The specific question one could ask, given that the $\mathrm{SU}(2)_{C}$ singlet from all $\left(\mathbf{N}_{L}, \mathbf{N}_{R}\right)$ multiplet has the same ratio of couplings to $W W$ and $Z Z$, is whether it is possible to distinguish the $\mathrm{SU}(2)_{C}$ singlet contained in a $\left(\mathbf{2}_{L}, \mathbf{2}_{R}\right)$ from that contained in a $\left(\mathbf{3}_{L}, \mathbf{3}_{R}\right)$. To this end we observe that the couplings, $g_{h_{1}^{0} W W}$ and $g_{h_{1}^{0} Z Z}$ in eqs. (3.16) and (3.17), and the gauge boson masses in eqs. (3.12) 
and (3.13) are given by two parameters: $N$ and the scalar VEV $v$. Solving for $v$ in terms of the masses and $N$ we obtain

$$
g_{h_{1}^{0} W W}=g_{h_{1}^{0} Z Z} c_{w}^{2}=\sqrt{\frac{N^{2}-1}{3}} g m_{W},
$$

Therefore the coupling becomes stronger as $N$ increases. The Higgs doublet has $N=$ 2, while the coupling of the $h_{1}^{0}$ in the $\left(\mathbf{N}_{L}, \mathbf{N}_{R}\right)$ is $\sqrt{\left(N^{2}-1\right) / 3}$ times larger than that in the Higgs doublet, resulting in a partial decay width that is $\left(N^{2}-1\right) / 3$ enhanced. Once $N$ is known, the complete $\mathrm{SU}(2)_{L} \times \mathrm{U}(1)_{Y}$ quantum number of the scalar resonance is determined.

As an example, at the LHC one could consider the production of the scalar in the vector boson fusion channels $W W / Z Z \rightarrow S \rightarrow W W$ and $W W / Z Z \rightarrow S \rightarrow Z Z$, which provide estimates of

$$
\left(\Gamma_{W W}+\Gamma_{Z Z}\right) \frac{\Gamma_{W W}}{\Gamma_{t}} \text { and }\left(\Gamma_{W W}+\Gamma_{Z Z}\right) \frac{\Gamma_{Z Z}}{\Gamma_{t}} .
$$

The total width $\Gamma_{t}$ could be extracted by measuring the Breit-Wigner shape of the invariant mass spectrum in the $Z Z$ channel. Then one could simply fit the partial widths $\Gamma_{W W}$ and $\Gamma_{Z Z}$ using the different hypothesis for $N$. Since the event rate in this case is proportional to $\Gamma_{W W / Z Z}^{2}$, if the total width remains the same the enhancement of a $N \geq 3$ multiplet over the Higgs doublet is $\left(N^{2}-1\right)^{2} / 9 \geq 64 / 9 \approx 7$, which is a significant enhancement.

\section{Discussion and outlook}

We have performed a general analysis up to dimension five of the couplings between electroweak vector boson pairs $V_{1} V_{2}$ and a Higgs look-alike $S$, assumed to be a neutral $C P$-even scalar resonance. We used the framework of unbroken custodial symmetry to group the possibilities into three "pure cases": scalars whose electroweak properties match a SM Higgs, scalars that are $\mathrm{SU}(2)_{L} \times \mathrm{SU}(2)_{R}$ singlets and thus couple to $V_{1} V_{2}$ only at dimension five, and scalars that couple to $V_{1} V_{2}$ as a 5 -plet under custodial $\mathrm{SU}(2)_{C}$.

Figure 1 shows that it should be straightforward to experimentally distinguish the 5-plet case from the SM-like case of a custodial singlet, using just the ratio of the $Z Z$ and $W W$ decay rates. Figure 2 illustrates that $\mathrm{SU}(2)_{L} \times \mathrm{SU}(2)_{R}$ singlets produce distinctive relations between the various ratios of $V_{1} V_{2}$ decay rates, emphasizing the importance of detecting all four decay channels: $W W, Z Z, \gamma \gamma$, and $Z \gamma$.

To implement our proposal one can either try to extract ratios of partial decay widths directly [28], or measure the individual partial decay widths into pairs of electroweak vector bosons first $[29,30]$ and then take the ratios. In the first possibility the event rate measured in each decay channel of a scalar resonance $S$ is given by

$$
B \sigma\left(V_{1} V_{2}\right)=\sigma(S) \times B r\left(S \rightarrow V_{1} V_{2}\right) .
$$

Therefore one could approximate the ratio of partial decay widths by the ratio of event rates in each channel, which are measured directly in collider experiments. It would be interesting to study ways to improve on the uncertainty arising from either possibilities. 
Since experimental analyses are often driven by final states observed, our study demonstrates the importance of having a correlated understanding of all decay channels into pairs of electroweak vector bosons to avoid misidentification. Tables I and II show how one can be badly fooled by measuring only two of the electroweak $V_{1} V_{2}$ decay channels for a candidate Higgs. The tables were generated from the predicted properties of a neutral $C P$-even spin 0 "Higgs" that is in fact an $\mathrm{SU}(2)_{L} \times \mathrm{SU}(2)_{R}$ singlet imposter. In table 1 the coefficients $\kappa_{1}, \kappa_{2}$ of the dimension-five operators in eq. (2.13) have been adjusted so that the ratio of branching fractions of $S \rightarrow Z Z$ over $S \rightarrow W W$ coincides with the SM value for the given masses $m_{S}$. In table II the same coefficients have been adjusted so that the branching ratio of $S \rightarrow \gamma \gamma$ over $S \rightarrow W W$ coincides with the SM value. In both cases measurement of the two remaining $V_{1} V_{2}$ decay rates unmasks the Higgs imposter in dramatic fashion.

In a real experiment, the analysis suggested here could be folded into hypothesis testing based on likelihood ratios designed to expose the spin and $C P$ properties of new heavy resonances [4, 5]. Higher order effects could be included, as well as the uncertainties associated with unfolding the experimental data to extract the $S \rightarrow V_{1} V_{2}$ production and decay properties.

\section{Acknowledgments}

We are grateful to Marcela Carena, Riccardo Rattazzi, and Maria Spiropulu for interesting discussions, and to Alvaro De Rújula for coining the phrase "Higgs imposters". I. L. was supported in part by the U.S. Department of Energy under contracts No. DE-AC0206CH11357 and No. DE-FG02-91ER40684. Fermilab is operated by the Fermi Research Alliance LLC under contract DE-AC02-07CH11359 with the U.S. Department of Energy.

\section{A General off-shell decay width}

We consider a massive spin-0 particle $S$ decaying to two off-shell vector bosons $V_{1}^{*}, V_{2}^{*}$. In the rest frame of $S$, and choosing the positive $z$-axis along the direction of $V_{2}$, the 4-momenta can be written:

$$
p_{S}=\left(m_{S}, 0,0,0\right) \quad p_{1}=m_{1}\left(\gamma_{1}, 0,0,-\beta_{1} \gamma_{1}\right), \quad p_{2}=m_{2}\left(\gamma_{2}, 0,0, \beta_{2} \gamma_{2}\right),
$$

where $m_{1}, m_{2}$ are the off-shell vector boson masses, and the boosts factors $\gamma_{1}, \gamma_{2}, \beta_{1}, \beta_{2}$ are defined by

$$
\begin{aligned}
\gamma_{1} & =\frac{m_{S}}{2 m_{1}}\left(1+\frac{m_{1}^{2}-m_{2}^{2}}{m_{S}^{2}}\right), \quad \gamma_{2}=\frac{m_{S}}{2 m_{2}}\left(1-\frac{m_{1}^{2}-m_{2}^{2}}{m_{S}^{2}}\right), \\
\beta_{1} \gamma_{1} & =\frac{m_{S}}{2 m_{1}} \sqrt{\left(1-\frac{\left(m_{1}+m_{2}\right)^{2}}{m_{S}^{2}}\right)\left(1-\frac{\left(m_{1}-m_{2}\right)^{2}}{m_{S}^{2}}\right)} \\
\beta_{2} \gamma_{2} & =\frac{m_{S}}{2 m_{2}} \sqrt{\left(1-\frac{\left(m_{1}+m_{2}\right)^{2}}{m_{S}^{2}}\right)\left(1-\frac{\left(m_{1}-m_{2}\right)^{2}}{m_{S}^{2}}\right)} .
\end{aligned}
$$

We will use the following convenient notation:

$$
\gamma_{a}=\gamma_{1} \gamma_{2}\left(1+\beta_{1} \beta_{2}\right)=\cosh \left(y_{2}-y_{1}\right), \quad \gamma_{b}=\gamma_{1} \gamma_{2}\left(\beta_{1}+\beta_{2}\right)=\sinh \left(y_{2}-y_{1}\right),
$$


where $y_{1}$ and $y_{2}$ are the vector boson rapidities, as well as the following useful identities:

$$
\gamma_{a}^{2}-\gamma_{b}^{2}=1, \quad \gamma_{a}=\frac{1}{2 m_{1} m_{2}}\left[m_{S}^{2}-\left(m_{1}^{2}+m_{2}^{2}\right)\right], \quad \gamma_{b}=\frac{m_{S}}{m_{1}} \beta_{2} \gamma_{2} .
$$

It is very convenient to compute the decay widths using helicity amplitudes. For this purpose we need to choose a consistent basis for the polarization vectors of the vector bosons:

$$
\begin{aligned}
& \epsilon_{2}\left(\lambda_{2}= \pm\right)= \pm \frac{1}{\sqrt{2}}(0,1, \pm i, 0), \quad \epsilon_{2}\left(\lambda_{2}=0\right)=\left(\beta_{2} \gamma_{2}, 0,0, \gamma_{2}\right) \\
& \epsilon_{1}\left(\lambda_{1}=\mp\right)= \pm \frac{1}{\sqrt{2}}(0,1, \pm i, 0), \quad \epsilon_{1}\left(\lambda_{1}=0\right)=\left(\beta_{1} \gamma_{1}, 0,0,-\gamma_{1}\right)
\end{aligned}
$$

where $\lambda_{1}, \lambda_{2}$ label the transverse and longitudinal polarizations.

Last but not least we will also need an expression for the two-body phase space:

$$
\begin{aligned}
d \Phi_{2}\left(p_{S} ; p_{1}, p_{2}\right) & =\frac{d^{3} p_{1} d^{3} p_{2}}{(2 \pi)^{3} 2 E_{1}(2 \pi)^{3} 2 E_{2}}(2 \pi)^{4} \delta^{4}\left(p_{S}-p_{1}-p_{2}\right) \\
& =\frac{1}{16 \pi^{2}} \frac{\left|\vec{p}_{1}\right|}{m_{S}} d \cos \theta d \phi
\end{aligned}
$$

where $\theta, \phi$ are the polar and azimuthal angles between the direction of $V_{2}$ and some other reference direction, e.g. the direction of the boost from the lab frame to the $S$ rest frame, or the direction of the beam. Note that

$$
\left|\vec{p}_{1}\right|=\left|\vec{p}_{2}\right|=m_{1} \beta_{1} \gamma_{1}=m_{2} \beta_{2} \gamma_{2}=\frac{m_{1} m_{2}}{m_{S}} \gamma_{b} .
$$

It is important to remember that when $V_{1}, V_{2}$ are distinguishable particles, we integrate $\theta, \phi$ over the full $4 \pi$ solid angle. However when $V_{1}, V_{2}$ are identical particles (e.g. two $Z$ 's or two $\gamma$ 's) we should only integrate $\theta$ from zero to $\pi / 2$, to avoid counting the same final state configuration twice. Thus the angular integration gives $2 \pi$ in this case, not $4 \pi$.

The differential off-shell decay width can be written:

$$
\frac{d^{2} \Gamma\left(S \rightarrow V_{1}^{*} V_{2}^{*}\right)}{d m_{1}^{2} d m_{2}^{2}}=\frac{2 \pi \delta_{V}}{2 m_{S}} \frac{m_{1} m_{2} \gamma_{b}}{16 \pi^{2} m_{S}^{2}} P_{1} P_{2} \sum_{\lambda_{1}, \lambda_{2}= \pm, 0}\left|\Gamma_{S V_{1} V_{2}}^{\mu \nu} \epsilon_{\mu}^{*}\left(\lambda_{1}\right) \epsilon_{\nu}^{*}\left(\lambda_{2}\right)\right|^{2}
$$

where $\delta_{V}=1$ for identical vector bosons and 2 otherwise. Here $\Gamma_{S V_{1} V_{2}}^{\mu \nu}$ is the $S V_{1} V_{2}$ coupling tensor that can be read off from the Lagrangian. The propagator factors

$$
P_{i}=\frac{M_{V_{i}} \Gamma_{V_{i}}}{\pi} \frac{1}{\left(m_{i}^{2}-M_{V_{i}}^{2}\right)^{2}+M_{V_{i}}^{2} \Gamma_{V_{i}}^{2}}
$$

become just $\delta\left(m_{i}^{2}-M_{V_{i}}^{2}\right)$ in the narrow width approximation. We will write the coupling tensor as

$$
\Gamma_{S V_{1} V_{2}}^{\mu \nu}=\left(\tilde{g}_{h V_{1} V_{2}}+\frac{\tilde{g}_{s V_{1} V_{2}}}{m_{S}} p_{1} \cdot p_{2}\right) g^{\mu \nu}-\frac{\tilde{g}_{s V_{1} V_{2}}}{m_{S}} p_{1}^{\nu} p_{2}^{\mu},
$$

where the coupling constants $g_{h}$ and $g_{s}$ are defined as coefficients of the following operators

$$
\frac{\delta_{V}}{2}\left(\tilde{g}_{h V_{1} V_{2}} S V_{1}^{\mu} V_{2 \mu}+\frac{\tilde{g}_{s V_{1} V_{2}}}{2 m_{S}} S V_{1}^{\mu \nu} V_{2 \mu \nu}\right) .
$$


In the standard model $\tilde{g}_{h V_{1} V_{2}}^{2}=8 m_{1}^{2} m_{2}^{2} G_{F} / \sqrt{2}$ for $W W$ and $Z Z$ channels and all other couplings vanish at the tree-level, while for an electroweak singlet scalar $\tilde{g}_{h V_{1} V_{2}}=0$. By angular momentum conservation the only nonvanishing contributions from the helicity sums are for $\left(\lambda_{1}, \lambda_{2}\right)=( \pm, \pm)$, or $(0,0)$ :

$$
\begin{aligned}
\sum_{\left(\lambda_{1}, \lambda_{2}\right)}\left|\Gamma^{\mu \nu} \epsilon_{\mu}^{*}\left(\lambda_{1}\right) \epsilon_{\nu}^{*}\left(\lambda_{2}\right)\right|^{2}= & \left|\tilde{g}_{h V_{1} V_{2}}\right|^{2}\left(2+\gamma_{a}^{2}\right)+\frac{m_{1}^{2} m_{2}^{2}}{m_{S}^{2}}\left|\tilde{g}_{s V_{1} V_{2}}\right|^{2}\left(2 \gamma_{a}^{2}+1\right) \\
& +\frac{6 m_{1} m_{2} \gamma_{a}}{m_{S}} \Re\left(\tilde{g}_{h V_{1} V_{2}} \tilde{g}_{s V_{1} V_{2}}^{*}\right),
\end{aligned}
$$

where $\Re(c)$ is the real part of the complex number $c$. Then the off-shell decay width is

$$
\begin{array}{r}
\frac{d \Gamma\left(S \rightarrow V_{1}^{*} V_{2}^{*}\right)}{d m_{1}^{2} d m_{2}^{2}}=\frac{2 \pi \delta_{V}}{2 m_{S}} \frac{m_{1} m_{2} \gamma_{b}}{16 \pi^{2} m_{S}^{2}}\left[\left|\tilde{g}_{h V_{1} V_{2}}\right|^{2}\left(2+\gamma_{a}^{2}\right)+\left|\tilde{g}_{s V_{1} V_{2}}\right|^{2} \frac{m_{1}^{2} m_{2}^{2}}{m_{S}^{2}}\left(2 \gamma_{a}^{2}+1\right)\right. \\
\left.+\Re\left(\tilde{g}_{h V_{1} V_{2}} \tilde{g}_{s V_{1} V_{2}}^{*}\right) \frac{6 m_{1} m_{2} \gamma_{a}}{m_{S}}\right] P_{1} P_{2}
\end{array}
$$

The total decay width of $S \rightarrow V_{1}^{*} V_{2}^{*}$ is given by

$$
\Gamma\left(S \rightarrow V_{1}^{*} V_{2}^{*}\right)=\int_{0}^{m_{S}^{2}} d m_{1}^{2} \int_{0}^{\left(m_{S}-\sqrt{m_{1}^{2}}\right)^{2}} d m_{2}^{2} \frac{d \Gamma\left(S \rightarrow V_{1}^{*} V_{2}^{*}\right)}{d m_{1}^{2} d m_{2}^{2}} .
$$

The above formula is valid even when the scalar mass crosses the mass thresholds of $W$ and $Z$ bosons. More explicitly, when both vector bosons are on-shell, $m_{1} \rightarrow m_{V}, m_{2} \rightarrow m_{V}$, we have

$$
\begin{aligned}
& \Gamma\left(S \rightarrow V_{1} V_{2}\right)= \frac{\delta_{V}}{32 \pi m_{S}} \sqrt{1-4 x}\left\{\left|\tilde{g}_{h V_{1} V_{2}}\right|^{2} \frac{1}{4 x^{2}}\left(1-4 x+12 x^{2}\right)\right. \\
&\left.+\left|\tilde{g}_{s V_{1} V_{2}}\right|^{2} \frac{m_{S}^{2}}{2}\left(1-4 x+6 x^{2}\right)+\Re\left(\tilde{g}_{h V_{1} V_{2}} \tilde{g}_{s V_{1} V_{2}}^{*}\right) 3 m_{S}(1-2 x)\right\} .
\end{aligned}
$$

For a standard model Higgs boson, $h$, we recover the well-known expression [24]

$$
\Gamma\left(h \rightarrow V_{1} V_{2}\right)=\delta_{V} \frac{G_{F}}{\sqrt{2}} \frac{m_{h}^{3}}{16 \pi} \sqrt{1-4 x}\left(1-4 x+12 x^{2}\right) .
$$

In the case of $S \rightarrow Z^{*} \gamma$, we have to take into account that only the transverse polarizations contribute, and take the limit $m_{2} \rightarrow 0$. As $m_{2} \rightarrow 0$

$$
\frac{d \Gamma\left(s \rightarrow Z^{*} \gamma\right)}{d m_{1}^{2}}=\frac{1}{32 \pi}\left|\tilde{g}_{s Z \gamma}\right|^{2} m_{S}\left(1-\frac{m_{1}^{2}}{m_{S}^{2}}\right)^{3} P_{1} .
$$

When the $Z$ is on-shell this becomes

$$
\Gamma(S \rightarrow Z \gamma)=\frac{1}{32 \pi}\left|\tilde{g}_{s Z \gamma}\right|^{2} m_{S}(1-x)^{3} .
$$

The width for $S \rightarrow \gamma \gamma$ follows from this (note we divide by 2 to get the correct phase space):

$$
\Gamma(S \rightarrow \gamma \gamma)=\frac{1}{64 \pi}\left|\tilde{g}_{s \gamma \gamma}\right|^{2} m_{S}
$$


Open Access. This article is distributed under the terms of the Creative Commons Attribution Noncommercial License which permits any noncommercial use, distribution, and reproduction in any medium, provided the original author(s) and source are credited.

\section{References}

[1] J.F. Gunion, H.E. Haber, G.L. Kane and S. Dawson, The Higgs hunter's guide, Frontiers in Physics series, Westview Press, U.S.A. (2000).

[2] A. Djouadi, The Anatomy of electro-weak symmetry breaking. I: the Higgs boson in the standard model, Phys. Rept. 457 (2008) 1 [hep-ph/0503172] [SPIRES].

[3] Q.-H. Cao, C.B. Jackson, W.-Y. Keung, I. Low and J. Shu, The Higgs mechanism and loop-induced decays of a scalar into two Z bosons, Phys. Rev. D 81 (2010) 015010 [arXiv: 0911.3398] [SPIRES].

[4] A. De Rujula, J. Lykken, M. Pierini, C. Rogan and M. Spiropulu, Higgs look-alikes at the LHC, Phys. Rev. D 82 (2010) 013003 [arXiv: 1001.5300] [SPIRES].

[5] Y. Gao et al., Spin determination of single-produced resonances at hadron colliders, Phys. Rev. D 81 (2010) 075022 [arXiv:1001.3396] [SPIRES].

[6] S.L. Adler, J.C. Collins and A. Duncan, Energy-momentum-tensor trace anomaly in spin $1 / 2$ quantum electrodynamics, Phys. Rev. D 15 (1977) 1712 [SPIRES].

[7] A. Djouadi, M. Spira and P.M. Zerwas, Two photon decay widths of Higgs particles, Phys. Lett. B 311 (1993) 255 [hep-ph/9305335] [SPIRES].

[8] W.D. Goldberger, B. Grinstein and W. Skiba, Light scalar at LHC: the Higgs or the dilaton?, Phys. Rev. Lett. 100 (2008) 111802 [arXiv:0708.1463] [SPIRES].

[9] Y. Bai, M. Carena and J. Lykken, Dilaton-assisted dark matter, Phys. Rev. Lett. 103 (2009) 261803 [arXiv:0909.1319] [SPIRES].

[10] T. Plehn, D.L. Rainwater and D. Zeppenfeld, Determining the structure of Higgs couplings at the LHC, Phys. Rev. Lett. 88 (2002) 051801 [hep-ph/0105325] [SPIRES].

[11] V. Hankele, G. Klamke, D. Zeppenfeld and T. Figy, Anomalous Higgs boson couplings in vector boson fusion at the CERN LHC, Phys. Rev. D 74 (2006) 095001 [hep-ph/0609075] [SPIRES].

[12] P. Sikivie, L. Susskind, M.B. Voloshin and V.I. Zakharov, Isospin breaking in technicolor models, Nucl. Phys. B 173 (1980) 189 [SPIRES].

[13] Particle Data Group collaboration, C. Amsler et al., Review of particle physics, Phys. Lett. B 667 (2008) 1 [SPIRES].

[14] R. Boughezal, J.B. Tausk and J.J. van der Bij, Three-loop electroweak correction to the $\rho$ parameter in the large Higgs mass limit, Nucl. Phys. B 713 (2005) 278 [hep-ph/0410216] [SPIRES].

[15] M. Awramik, M. Czakon and A. Freitas, Electroweak two-loop corrections to the effective weak mixing angle, JHEP 11 (2006) 048 [hep-ph/0608099] [SPIRES].

[16] H. S. Tsao, Higgs boson quantum numbers and the Pell equation, in the proceedings of the 1980 Guangzhou Conference on Theoretical Particle Physics, H. Ning and T. Hung-yuan eds., Science Press, Beijing China (1980). 
[17] H.E. Haber and H.E. Logan, Radiative corrections to the $Z b \bar{b}$ vertex and constraints on extended Higgs sectors, Phys. Rev. D 62 (2000) 015011 [hep-ph/9909335] [SPIRES].

[18] J.R. Ellis, M.K. Gaillard and D.V. Nanopoulos, A phenomenological profile of the Higgs boson, Nucl. Phys. B 106 (1976) 292 [SPIRES].

[19] M.A. Shifman, A.I. Vainshtein, M.B. Voloshin and V.I. Zakharov, Low-energy theorems for higgs boson couplings to photons, Sov. J. Nucl. Phys. 30 (1979) 711 [Yad. Fiz. 30 (1979) 1368] [SPIRES].

[20] I. Low, R. Rattazzi and A. Vichi, Theoretical constraints on the Higgs effective couplings, JHEP 04 (2010) 126 [arXiv:0907.5413] [SPIRES].

[21] H. Georgi and M. Machacek, Doubly charged Higgs bosons, Nucl. Phys. B 262 (1985) 463 [SPIRES].

[22] M.S. Chanowitz and M. Golden, Higgs boson triplets with $M_{W}=M_{Z} \cos \theta \omega$, Phys. Lett. B 165 (1985) 105 [SPIRES].

[23] J.F. Gunion, R. Vega and J. Wudka, Higgs triplets in the standard model, Phys. Rev. D 42 (1990) 1673 [SPIRES].

[24] B.W. Lee, C. Quigg and H.B. Thacker, Weak interactions at very high-energies: the role of the Higgs boson mass, Phys. Rev. D 16 (1977) 1519 [SPIRES].

[25] T.G. Rizzo, Decays of heavy Higgs bosons, Phys. Rev. D 22 (1980) 722 [SPIRES].

[26] W.-Y. Keung and W.J. Marciano, Higgs scalar decays: $H \rightarrow W^{ \pm} X$, Phys. Rev. D 30 (1984) 248 [SPIRES].

[27] A. Grau, G. Panchieri and R.J.N. Phillips, Contributions of off-shell top quarks to decay processes, Phys. Lett. B 251 (1990) 293 [SPIRES].

[28] D. Zeppenfeld, Higgs couplings at the $L H C$, in the proceedings of the APS/DPF/DPB Summer Study on the Future of Particle Physics (Snowmass2001), June 30-July 21, Snowmass, Colorado, U.S.A. (2001), hep-ph/0203123 [SPIRES].

[29] M. Dührssen et al., Extracting Higgs boson couplings from LHC data, Phys. Rev. D 70 (2004) 113009 [hep-ph/0406323] [SPIRES].

[30] R. Lafaye, T. Plehn, M. Rauch, D. Zerwas and M. Dührssen, Measuring the Higgs Sector, JHEP 08 (2009) 009 [arXiv:0904.3866] [SPIRES]. 